\title{
Persistent Organic Pollutants and Type 2 Diabetes: A Critical Review of Review Articles
}

\section{OPEN ACCESS}

Edited by:

Angel Nadal,

Universidad Miguel Hernández de Elche, Spain

Reviewed by:

German Cano-Sancho, INRA Centre Angers-Nantes Pays de la Loire, France

Michele Andrea La Merrill, University of California, Davis, United States

*Correspondence: Duk-Hee Lee lee_dh@knu.ac.kr

Specialty section: This article was submitted to

Systems and Translational Endocrinology,

a section of the journal

Frontiers in Endocrinology

Received: 31 July 2018 Accepted: 12 November 2018 Published: 27 November 2018

Citation:

Lee Y-M, Jacobs DR Jr and Lee D-H (2018) Persistent Organic Pollutants and Type 2 Diabetes: A Critical Review of Review Articles.

Front. Endocrinol. 9:712. doi: 10.3389/fendo.2018.00712

\begin{abstract}
Yu-Mi Lee ${ }^{1}$, David R. Jacobs Jr. ${ }^{2}$ and Duk-Hee Lee ${ }^{1,3 *}$
${ }^{1}$ Department of Preventive Medicine, School of Medicine, Kyungpook National University, Daegu, South Korea, ${ }^{2}$ Division of Epidemiology and Community Health, School of Public Health, University of Minnesota, Minneapolis, MN, United States, ${ }^{3}$ BK21 Plus KNU Biomedical Convergence Program, Department of Biomedical Science, Kyungpook National University, Daegu, South Korea
\end{abstract}

Low dose persistent organic pollutants (POPs) have emerged as a new risk for type 2 diabetes (T2D). Despite substantial evidence from human and experimental studies, there are several critical issues which have not been properly addressed by POPS researchers. First, as POPs exist as mixtures, findings about POPs from human studies should be interpreted from the viewpoint of lipophilic chemical mixtures which include both measured and unmeasured POPs. Second, as POPs can directly reduce insulin secretion of beta cells, the role of POPs may be more prominent in the development of beta-cell dysfunction-dominant T2D rather than insulin resistance-dominant T2D. Third, there are multidimensional interrelationships between POPs and adipose tissue. Even though POPs are now considered as a new risk factor for T2D, independent of obesity, POPs and obesity are mechanistically linked to each other. POPs are involved in key mechanisms linking obesity and T2D, such as chronic inflammation of adipose tissue and lipotoxicity with ectopic fat accumulation. Also, POPs can explain puzzling human findings which suggest benefits of obesity because healthy adipose tissue can be protective by reducing the amount of POPs reaching other organs. Fourth, non-linear dose-response relationships between POPs and T2D are biologically possible. Although POPs are well-known endocrine disrupting chemicals (EDCs), mitochondrial dysfunction may be a more plausible mechanism due to unpredictability of EDC mixtures. As adipose tissue plays a role as an internal exposure source of POPs, how to manage POPs inside us may be essential to protect against harms of POPs.

Keywords: chemical mixtures, diabetes, insulin resistance, obesity, organochlorine pesticides, persistent organic pollutants, polychlorinated biphenyls

\section{INTRODUCTION}

Persistent organic pollutants (POPs) include a wide range of organic compounds which are resistant to degradation by chemical or biological processes. As a result, they are highly persistent in the environment and bio-accumulate in living organisms. Typical examples are chlorinated POPs such as organochlorine pesticides (OCPs), polychlorinated biphenyls (PCBs), or dioxins, brominated POPs such as polybrominated diphenyl ethers, or fluorinated POPs such as perfluoroalkyl and polyfluoroalkyl substances.

Although toxicity of high dose POPs is well-known, the recent concern is the possibility of adverse effects of low dose POPs, similar to the current environmental exposure levels. Among 
many diseases suspected to be linked to low dose POPs, type 2 diabetes (T2D) is the most convincing, given substantial evidence from both epidemiological and experimental studies.

Since 2010, we identified nine published review articles about human studies on POPs and T2D (1-9). Therefore, rather than adding one more ordinary review article on the same topic, this narrative review was written to bring up several provocative issues which are commonly missed by epidemiologists on POPs and $\mathrm{T} 2 \mathrm{D}$.

First, we briefly summarize the nine published review articles on POPs and T2D based on their own conclusions, rather than presenting our own quantitative analyses. Then we discuss (1) the importance of POPs as mixtures, (2) the role of POPs in inducing beta-cell dysfunction, (3) multi-dimensional interrelationships between POPs and adipose tissue, and (4) non-linear dose response relationships. Consideration of these aspects leads us to debate why currently prevailing actions (including regulations and suggestions for avoidance of POPs) focused on individual chemicals are not effective in protecting the public from POPsrelated risk.

In this review, the focus is low dose environmental exposure, not occupational or accidental high dose exposure. Also, among various POP compounds, we deal with chlorinated POPs because they are ones which have shown the most consistent results in human studies. The evidence from brominated or fluorinated POPs is weak and inconsistent (9). Thus, unless we specify brominated or fluorinated, POPs means chlorinated POPs in this review.

\section{A BRIEF SUMMARY OF REVIEW ARTICLES ON HUMAN STUDIES ON POPS AND T2D}

Table 1 summarizes conclusions from review articles about POPs and T2D which were published since 2010 (1-9). Sentences in the "Conclusion" column were extracted from abstract, highlights, and main findings without any modification. Some reviews focused on POPs while others covered a wide range of endocrine disrupting chemicals (EDCs; POPs were considered to be EDCs).

Although they include systemic reviews, meta-analyses, and narrative reviews, all reviews concluded that there is potentially a role of POPs in the development of T2D despite some inconsistencies and research gaps. However, there are several critical issues which have not been properly considered in most original articles and reviews about POPs and T2D. As they can substantially influence the establishment of causality, the discussion of these issues can be helpful to understand the findings from published articles and plan future epidemiological studies.

\section{CRITICAL ISSUES FOR HUMAN STUDIES OF POPs AND T2D \\ POPS as a Surrogate Marker of Lipophilic Chemical Mixtures}

Most human studies about POPs and T2D have adopted individual chemical-based analyses. Namely, they measured
TABLE 1 | Summary of review articles about epidemiological studies of persistent organic pollutants (POPs) and type 2 diabetes (T2D).

\begin{tabular}{|c|c|c|}
\hline Authors & $\begin{array}{l}\text { Publication } \\
\text { year }\end{array}$ & Verbatim conclusion from each review \\
\hline Taylor et al. (1) & 2013 & $\begin{array}{l}\text { The overall evidence is sufficient for a positive } \\
\text { association of some organochlorine POPs with } \\
\text { type } 2 \text { diabetes. }\end{array}$ \\
\hline Wu et al. (2) & 2013 & $\begin{array}{l}\text { These findings support an association between } \\
\text { POP exposure and the risk of T2D. }\end{array}$ \\
\hline Lee et al. (3) & 2014 & $\begin{array}{l}\text { The evidence as a whole suggests that, rather } \\
\text { than a few individual POPs, background } \\
\text { exposure to POP mixtures-including } \\
\text { organochlorine pesticides and polychlorinated } \\
\text { biphenyls-can increase T2D risk in humans. }\end{array}$ \\
\hline Magliano et al. (4) & 2014 & $\begin{array}{l}\text { In summary, while the overall evidence is } \\
\text { strongly suggestive of an independent } \\
\text { relationship between POPs and diabetes, } \\
\text { some inconsistencies exist. }\end{array}$ \\
\hline Ngwa et al. (5) & 2015 & $\begin{array}{l}\text { Despite different levels of risk in prospective } \\
\text { studies and inconsistent results, the causal } \\
\text { effect of POPs on diabetes is supported by } \\
\text { in-vitro and in-vivo experimental studies. }\end{array}$ \\
\hline Jaacks et al. (6) & 2015 & $\begin{array}{l}\text { The literature suggests a positive association } \\
\text { between select POPs and diabetes. }\end{array}$ \\
\hline Song et al. (7) & 2016 & $\begin{array}{l}\text { Serum concentrations of persistent EDCs* } \\
\text { were significantly associated with T2D risk. }\end{array}$ \\
\hline Evangelou et al. (8) & 2016 & $\begin{array}{l}\text { Data suggest an association between } \\
\text { organochlorine exposure and type } 2 \text { diabetes }\end{array}$ \\
\hline Lind et al. (9) & 2018 & $\begin{array}{l}\text { Evidence is accumulating that EDCs* might be } \\
\text { involved in diabetes development. Best } \\
\text { evidence exists for p,p'-DDE. }\end{array}$ \\
\hline
\end{tabular}

"EDCs (endocrine disrupting chemicals), POPs are classified as EDCs.

serum concentrations of several or dozens of compounds belonging to POPs, evaluated individual associations between specific compounds and $\mathrm{T} 2 \mathrm{D}$, and interpreted their results focusing on those specific compounds which showed statistical significance. As a result, most reviews also followed the same strategy. In some reviews, only specific compounds such as hexachlorobenzene, p,p'-DDE, and PCBs, but not others, were considered to be linked to the risk of T2D $(2,9)$.

However, this approach should be reconsidered because recent human studies about POPs and T2D have been performed among general populations. It is well-known that there are substantial positive correlations among serum concentrations of various POP compounds $(10,11)$. Therefore, the epidemiologic findings on POPs should not be interpreted from the viewpoint of individual compounds which were directly measured and demonstrated statistical significance. The key feature of environmental exposure to POPs is the chronic exposure to the mixture of a variety of lipophilic chemicals at low dose even though absolute concentrations of individual compounds are variable. Focusing only on several compounds can largely distort the whole picture.

In most epidemiological studies, serum concentrations of POPs have been used as an exposure marker of POPs. Although concentrations of most synthetic chemicals measured in blood or 
urine are indicators of the current or recent exposure levels from the environment, serum concentrations of POPs are different. Humans are exposed to POPs through external exposure sources such as POPs-contaminated food. However, once POPs enter the body, they are primarily stored in adipose tissue and slowly released into the circulation to be eliminated over several years (12). Therefore, ultimately serum concentrations of POPs are largely determined by (1) the amount of POPs released from adipose tissue to circulation and (2) the amount of POPs eliminated from circulation.

In this situation, the findings about POPs in human studies should be interpreted beyond the compounds which are directly measured in each study. Measured POPs should be considered as surrogate markers of lipophilic chemical mixtures which include measured POPs, but also include unmeasured ones. This point has an important implication regarding how to approach the issue of POPs to protect the public. For example, even if we succeeded in completely eliminating specific POPs, this may not lead to the decrease of POPs-related disease because we have done nothing with other lipophilic chemicals which coexist with the eliminated POPs in the mixture. This aspect of POPs suggests that the most effective public health action to reduce harms from POPs should target lipophilic chemical mixtures.

\section{Role of POPs in Inducing Beta-Cell Dysfunction}

T2D is increasingly recognized as a heterogeneous condition, ranging from predominantly insulin resistance with relative insulin deficiency to predominantly an insulin secretory defect with insulin resistance (13). Although most epidemiological studies about POPs have evaluated T2D as a whole, the role of POPs may differ depending on subtypes of T2D.

Obesity is the most important risk factor for insulin resistance-dominant T2D, however the role of adiposity is weak in T2D preceded predominantly by beta-cell dysfunction (14). Genetic predisposition has been considered a key determinant of beta-cell function (15), but the role of genes is still largely unknown despite many genome-wide association studies(16).

Environmental chemicals such as POPs can play a role in the development of beta-cell dysfunction (17). Although animal experimental studies have reported that the exposure to POPs can induce insulin resistance $(18,19)$, human studies evaluating both insulin resistance and insulin secretion have reported that serum concentrations of POPs were more strongly associated with decreased insulin secretion than with increased insulin resistance (20-23). An in-vitro cell study demonstrated that POPs can directly reduce insulin secretion at very low dose such as 1 $\mathrm{pmol} / \mathrm{L}$ (23).

As decreased insulin secretion is a necessary step in developing both types of T2D, POPs can explain both types of T2D. However, the role of POPs may be more salient in beta-cell dysfunction-dominant $\mathrm{T} 2 \mathrm{D}$ than insulin resistance-dominant T2D because the overproduction of insulin by pancreatic betacells during insulin resistance can mask the direct effect of POPs on beta-cell function (23). Also, POPs can explain why betacell dysfunction-dominant T2D is common in Asian and elderly people (24-26) who tend to have high serum concentrations of OCPs $(27,28)$.

\section{Multi-Dimensional Aspects of Interrelationships Between POPs and Adipose Tissue}

POPs have been evaluated as a new risk factor for T2D, independent of traditional risk factors for T2D such as obesity or lack of physical activity. As a result, in epidemiological studies of POPs and T2D, obesity has been considered as a confounder. However, obesity cannot be a simple confounder in the relationship between POPs and T2D. The role of POPs should be comprehensively evaluated and interpreted, considering possibly interactive roles with obesity, due to their innate interrelationship.

Under the current paradigm, obesity is a key risk factor for many insulin resistance-related diseases such as T2D. Several mechanisms explain how obesity can increase these diseases. First, obesity can induce chronic inflammation of adipose tissue and release pro-inflammatory cytokines (29). Second, obesity increases the release of free fatty acid to circulation and promotes fat deposits in ectopic sites such as liver, muscle, and pancreas (30).

However, POPs may participate in all these mechanisms, explained in detail below. Also, POPs can explain some puzzling findings on obesity which cannot be explained by the current paradigm of obesity. We present five different possible dimensions linking POPs and adipose tissue (Figure 1). Among them, dimensions 1 and 2 show that POPs can explain traditional obesity-related harmful effects while dimensions 3 and 4 show that POPs can explain puzzling findings on obesity. Dimension 5 casts a question on the current prevailing viewpoint which directly links obesogen-inducing chemicals to diabetesinducing chemicals. All these issues are critical to understand the role of POPs in humans in a more comprehensive way and also to contrive effective ways to protect the public from POPs.

\section{Dimension 1: POPs in Adipose Tissue Can Induce Inflammation in Adipose Tissue}

Adipose tissue is not only a reservoir of chronic internal POPs exposure, but also a possible tissue pathologic target of POPs (31). In-vivo and in-vitro experimental studies reported that low dose POPs can induce pro-inflammatory change in adipose tissue $(18,19,31-34)$. Importantly, POPs-induced inflammation is possible regardless of obesity (19).

A topic for future studies is the interesting speculation that obesity can exacerbate the inflammatory effects of POPs. For example, if POPs are released from lipid droplets of adipocytes to interstitial spaces through necrosis of the hypertrophic adipocytes which are common among obese persons (35), the existence of poorly degradable foreign bodies such as POPs can trigger an adipose tissue immune response. 


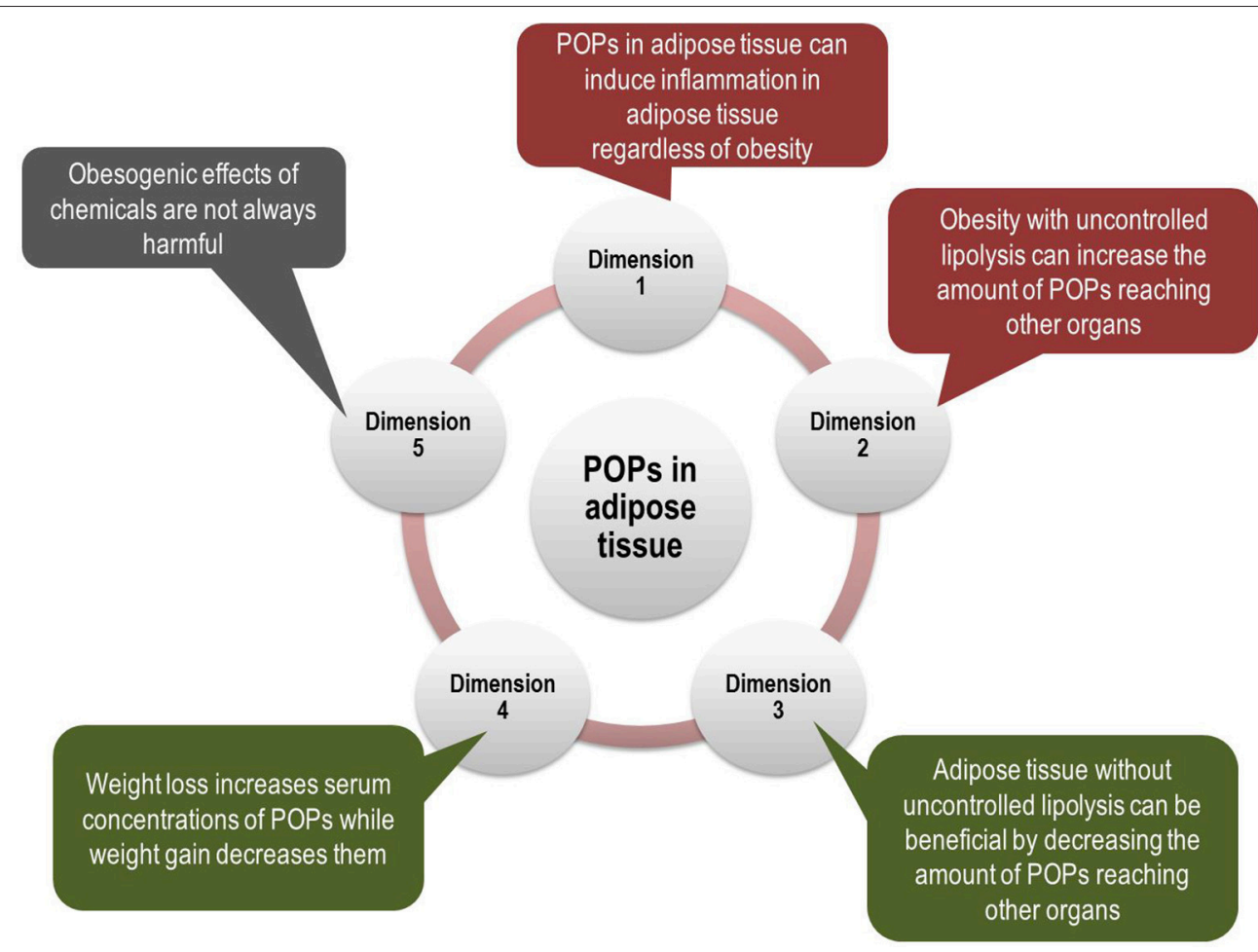

FIGURE 1 | Multi-dimensional aspects of interrelationships between persistent organic pollutants (POPs) and adipose tissue. Even though POPs have been evaluated as a new risk factor for type 2 diabetes (T2D), independent of obesity, the role of POPs should be evaluated together with obesity due to their innate interrelationship. POPs are involved in key mechanisms linking obesity and T2D such as pro-inflammatory adipose tissue and ectopic fat accumulation (Dimensions 1 and 2). In addition, POPs can explain puzzling findings about obesity, which suggest beneficial effects of adipose tissue (Dimensions 3 and 4). Finally, obesogenic effects of chemicals may not always be harmful (Dimension 5). All these issues are critical to understand the role of POPs in the development of T2D.

\section{Dimension 2: Obesity With Uncontrolled Lipolysis Can Increase the Amount of POPs Reaching Other Organs}

At normal physiological conditions, lipolysis rates of adipose tissue are tightly regulated through hormonal signals depending on caloric need (36). However, among obese persons, uncontrolled lipolysis is common and increased lipolysis accelerates the efflux of free fatty acids from adipose tissue to the circulation and ectopic fat accumulation (37). Insulin resistance can further enhance lipolysis in adipocytes (38).

Importantly, POPs are also released from adipocytes to circulation during lipid mobilization (39) and POPs in circulation can easily reach other organs (40). Although on average there is a higher risk of uncontrolled lipolysis among obese persons than lean persons, even in non-obese persons, the release of POPs to circulation can increase if uncontrolled lipolysis exists. Toxicokinetics of POPs during lipolysis should be considered in all mechanisms related to lipolysis of adipose tissue.

\section{Dimension 3: Adipose Tissue Without Uncontrolled Lipolysis Can Be Beneficial by Decreasing the Amount of POPs Reaching Other Organs}

In contrast to Dimension 2, as far as adipocyte function is physiologically healthy without uncontrolled lipolysis is concerned, greater adipose tissue can be more advantageous than less adipose tissue. The reason is that the storage of POPs in adipose tissue can reduce the amount of POPs reaching other organs (41) even though the storage of POPs in adipose tissue can have a negative side effect such as increasing half-lives of POPs in the long-term.

This aspect of adipose tissue may be helpful in explaining the obesity paradox (42): a better survival among overweight or obese patients or elderly despite a role of obesity as an important risk factor for many chronic diseases. The obesity paradox is also observed among patients with T2D, the exemplary obesity-related disease (43). Although several mechanisms such as body composition, cardiorespiratory fitness, or nutritional status have been suggested as possible explanations for the obesity paradox (42), POPs can provide a better explanation because the beneficial role of adipose tissue may be more important in patients or elderly than in healthy young persons. General features of patients with chronic diseases or elderly are the disturbance of homeostasis. In these conditions, the safe storage of pollutants in adipose tissue can be crucial. Supporting this speculation, there is a human study which showed that the beneficial role of adipose tissue may be increasing as serum concentration of POPs increases (44). 


\section{Dimension 4: Weight Loss Increases Serum Concentrations of POPs While Weight Gain Decreases Them}

In Dimension 2, we discussed that obesity can increase the release of POPs from adipose tissue to circulation through uncontrolled lipolysis. Ironically, weight loss also increases serum POPs concentrations $(45,46)$. On the other hand, weight gain can decrease them by sequestering POPs from the circulation into adipose tissue $(40,47)$. Therefore, possible effects due to dynamics of POPs during weight change are contrary to conventional expected effects of weight change from the viewpoint of fat mass. In that viewpoint, intentional weight loss is beneficial while weight gain is harmful.

Even though the improvement of metabolic risks with weight loss is well-known, the toxicokinetics of POPs during weight loss can negatively affect resting metabolic rate, thyroid hormone levels, and the oxidative potential of skeletal muscle $(45,48,49)$. Also, it can be helpful to explain why the intensive lifestyle intervention focusing on weight loss among overweight or obese patients with T2D failed to show a decrease of the development of cardiovascular diseases, compared to the usual management group (50). Repeated experience of weight loss and gain would be the most serious concern due to tissue redistribution of POPs between adipose tissue and other organs (40).

However, the different release pattern of POPs between Dimension 2 and Dimension 4 warrants further discussion. The release of POPs among obese persons through uncontrolled lipolysis is chronic and subtle while the release of POPs during intentional weight loss is temporary and large. Compared to the release of a large amount of POPs for a short period time, the release of a small amount of POPs for a long period can be more harmful because POPs-related health effects do not seem to increase linearly with increasing dose of POPs, as discussed below. In addition, exercise and changes in diet habit which are commonly accompanied with intentional weight loss can increase the elimination of POPs (51) and mitigate possible harms of POPs (52).

Importantly, even though it is intentional, weight loss among the elderly can be problematic. Elderly people can release more POPs into circulation than younger people because of more contamination of adipose tissue with POPs (27), but the capability of metabolizing and excreting xenobiotics is decreasing with aging (53). Therefore, POPs in circulation in the elderly can more easily reach other lipid-rich organs such as the brain. Recently, the dynamics of POPs has been suggested as a key factor to explain a high risk of dementia among patients with T2D as well as puzzling findings on obesity and dementia (54).

\section{Dimension 5: Obesogenic Effects of Chemicals May Not Always Be Harmful}

Recently, obesity-inducing effects of environmental chemicals including POPs have gained attention from researchers (55). Obesogens typically act at environmentally relevant doses during critical windows of prenatal or postnatal development to promote obesity later in life and these effects can be transmitted to their descendants (56). Possible mechanisms include increasing number of fat cells, increasing size of fat cells, and modulating hormones affecting appetite, satiety, and energy metabolism (57).

The current prevailing viewpoint on obesogens is that they can also act as diabetogens because they contribute to the development of obesity (58). However, the role of adipose tissue to protect other organs from POPs (Dimension 3) should be incorporated in interpreting the implication of obesogens. Among two mechanisms of the adipose tissue expansion, hypertrophy-dominant obesity (increase in cell size) is harmful due to pro-inflammatory cytokine release and impaired insulin sensitivity, but hyperplasia-dominant obesity (increase in cell number) is known to be beneficial (59). One of the key mechanisms by which obesogens promote adiposity is by increasing adipogenesis (56). Therefore, as far as they can provide healthy adipose tissue, their adipogenesis-promoting effect is not in itself necessarily harmful.

Although the obesogenic effect of DDT and their metabolites have been reported in human, in-vivo, and in-vitro studies (60), some POPs can suppress adipogenesis $(33,61)$. From the viewpoint of Dimension 3, these chemicals can be more harmful than obesogens if they diminish the safe storage site for lipophilic chemicals. The reality is much more complicated. For example, the same chemicals can increase adipogenesis at low dose while they can inhibit adipocyte differentiation at high dose (32). Also, there are synergic or antagonistic effects on adipogenesis based on studies of only two chemicals (33). Therefore, it would be almost impossible to predict what will be the net effect of chemical mixtures on the development of obesity.

As we discussed in Dimension 1, adipocytes were observed to be in a pro-inflammatory state regardless of whether POPs induced adipogenesis or reduced adipogenesis $(32,33)$. Therefore, rather than linking POPs to obesogens, investigation of direct harmful effects of POPs on adipose tissue and other organs would be more worthwhile in future research.

\section{Non-linearity}

Body burden of OCPs and PCBs in current general populations are much lower than they were in the era when these chemicals were actively used. Then, why do current low dose POPs reveal significant associations in human studies?

Interestingly, risks of many common environmental chemicals do not increase linearly within the range of environmental low dose exposure (62-64). POPs have also demonstrated the possibility of non-linearity with T2D (3). Within the low dose range, the risk of disease increases linearly within the relatively low dose range, but it does not further increase as dose increases. It can slow down, flatten, or even decrease with increasing doses. This feature is different from the linear dose-response relationship which is commonly observed in the range of high dose toxicity.

This non-linearity has been considered to be biologically implausible and was criticized as artifactual, arising from confounding or bias $(65,66)$. However, the non-linearity is plausible through several mechanisms. First, if chemicals are harmful through endocrine disruption, they can show nonlinearity (67). Second, the activation of stress responses with certain levels of chemicals, called hormesis, can be another 
mechanism to explain non-linearity (68). The traditional concept of hormesis does not consider possible harmful effects at subhormetic very low dose, but only contrasts "beneficial low dose zone" vs. "toxic high dose zone" (69). Within the environmental exposure range, the non-linearity can be the result of the combination of a "sub-hormetic harmful zone" and a "hormetic beneficial zone."

Epidemiologists should understand how non-linearity can affect human studies. Historically, populations with high dose exposure to chemicals have been considered as optimal for investigation of the association between chemical exposure and outcomes. Under non-linearity, however, the association between any exposure and disease should be studied among populations with low dose exposure because the exposure range would correspond to the low dose, linear part of the dose-response relationship. In populations with relatively high dose exposure, the observed result may well be a null association because the exposure range would be in the flattened, higher dose part of the dose-response relationship. This kind of non-linearity can be one reason why current general populations with low body burden of OCPs and PCBs show clear results.

Under non-linearity, conventional quantitative methods of reviews such as meta-analyses or pooled-analyses can be problematic because they assume linearity when summarizing results such as relative risks or odds ratios from individual studies which have been performed in various populations with different levels of POPs.

\section{POPS AS MITOCHONDRIAL TOXIN, NOT CONVENTIONAL EDCs, CAN BETTER EXPLAIN HUMAN FINDINGS ON POPS AND T2D}

POPs are well-known EDCs and T2D is an endocrine disease. Therefore, it is common to consider that POPs would be linked to T2D as EDCs, as seen in the titles of some review articles $(7,9)$. However, it may be difficult to explain human findings about POPs and T2D by the endocrine disrupting properties of POPs.

Several EDCs acting on the same pathway, such as estrogenic chemicals, have gained a lot of attention from researchers due to strong synergic effects (70). However, the unpredictable antagonistic effects of EDCs acting on different pathways are largely ignored (71-74). For example, the mixture of even two different EDCs (estrogenic and androgenic), did not produce predictable mixture effects. POPs are the mixture of diverse EDCs acting as either agonists or antagonists on different hormone receptors which can engage in crosstalk with each other. Therefore, it would be impossible to reliably evaluate the net effect of POPs acting as EDCs in humans.

Considering the complexity of EDC mixtures, other mechanisms might be more plausible as an explanation for the consistent findings about POPs relating to T2D in human studies. In fact, many environmental chemicals are known as mitochondrial toxins (75). Evidence is accumulating that low dose POPs can induce mitochondrial dysfunction and/or reduce oxidative phosphorylation capacities $(18,76-78)$. Although high dose POPs are well-known direct mitochondrial toxins (79), recent studies highlight functional impairment of mitochondria by low dose POPs. In fact, endocrine disruption can occur as a result of mitochondrial dysfunction because mitochondria are essential sites for steroid hormones biosynthesis in the steroidogenic cells (80).

Mitochondrial dysfunction is currently linked to many common age-related chronic diseases including T2D (81). However, the functional impairment of mitochondria can be reversed by restoration of their function. In the section "Nonlinearity," we discussed hormetic stress responses as a mechanism to explain the flattened part of the dose-response curve in the higher part of the low dose range. The activation of mitochondrial function is a typical example of hormetic stress response (82). Therefore, the non-linearity observed in the association between POPs and T2D can be explained by dynamics of mitochondrial function.

Particularly, it is important to note that there are health behaviors which can improve mitochondrial function (83). They include exercise, calorie restriction, and intake of phytochemicals in plant food (84-86). At present, regardless of POPs, these health behaviors are known as beneficial to decrease the risk of T2D and glycemic control among T2D patients. However, POPs may be a piece of the puzzle which has been missed in the relation between healthy behaviors and T2D.

\section{PERSPECTIVE ON FUTURE STUDIES OF POPs}

At present, researchers tend to think that even a small increased risk of T2D among persons due to POPs will have huge public health impact because of the ubiquitous presence of POPs and the high prevalence of T2D. However, as issues related to POPs cannot be separated from obesity issues, as we discussed above, a more comprehensive viewpoint about POPs is needed.

As the majority of published human studies about POPs and T2D are still cross-sectional, more prospective studies on POPs and T2D would be desirable. The role of POPs can be better studied among populations in which beta-cell dysfunctiondominant T2D is common, such as Asians or the elderly. In particular, the relationship between POPs and obesity on the development of T2D should be thoroughly investigated. However, the value of long-term follow-up may not be as good as epidemiologists generally believe for reasons discussed below.

First, as the dynamics of adipose tissue continuously affect serum concentration of POPs, the baseline POP value may become less representative of POPs exposure as the follow-up period gets longer. In modern society, many persons repeatedly experience weight gain and weight loss. This experience would be more frequent among obese persons, a high risk group for T2D. Therefore, although POPs are an example of synthetic chemicals with reliable exposure assessment due to their long half-lives (87), the value of baseline serum concentrations of POP decreases as follow-up period gets longer. 
Second, in the case of exposure variables such as POPs which are expensive to measure, a nested case control study is generally regarded as the best design for human studies. However, as the value of measurement of POPs at baseline is decreased when the follow-up period increases for the reason we discussed above, the value of a nested case control study is also limited. Additionally, serum stored in periods when POPs were actively used would not be advantageous due to their high serum concentrations among study subjects. Under non-linearity, populations with low dose exposure are better to explore the relationship between exposure and disease than are populations with high dose exposure.

Third, the effects of POPs on the development of T2D may be dynamic. Unlike high dose toxicity with irreversible damage, functional impairment due to low dose exposure can be reversible. For example, as we discussed above, healthy lifestyles can reverse harmful effects of POPs through the improvement of mitochondrial function. Therefore, the interaction between healthy behaviors and POPs can dynamically influence the development of T2D.

All these issues suggest that a prospective study or a nested case control study with only baseline information may fail to uncover a role of POPs in the development of T2D. This problem becomes more serious as the follow-up period gets longer. Considering the necessity of repeated measurements of POPs, obesity, and other relevant variables, epidemiologists need to plan a reasonable follow-up period. The development of cheap and quick bioassay methods to assess POPs in bio-specimens would be useful for large-scale human studies with repeated measurements.

Considering the difficulties in human studies, laboratory studies mimicking human exposure would be crucial. For this purpose, animal models using lipolysis of adipose tissue such as hypertrophic adipocytes with uncontrolled lipolysis or repeated weight cycling can be useful. Mitochondrial dysfunction by the environmentally-relevant doses of POPs and the possibility of their restoration by the activation of mitohormesis would be another important study topic in the field of laboratory research.

\section{REFERENCES}

1. Taylor KW, Novak RF, Anderson HA, Birnbaum LS, Blystone C, Devito $\mathrm{M}$, et al. Evaluation of the association between persistent organic pollutants (POPs) and diabetes in epidemiological studies: a national toxicology program workshop review. Environ Health Perspect. (2013) 121:774-83. doi: 10.1289/ehp. 1205502

2. Wu H, Bertrand KA, Choi AL, Hu FB, Laden F, Grandjean P, et al. Persistent organic pollutants and type 2 diabetes: a prospective analysis in the nurses' health study and meta-analysis. Environ Health Perspect. (2013) 121:153-61. doi: 10.1289/ehp.1205248

3. Lee DH, Porta M, Jacobs DR Jr, Vandenberg LN. Chlorinated persistent organic pollutants, obesity, and type 2 diabetes. Endocr Rev. (2014) 35:557601. doi: 10.1210/er.2013-1084

4. Magliano DJ, Loh VH, Harding JL, Botton J, Shaw JE. Persistent organic pollutants and diabetes: a review of the epidemiological evidence. Diabetes Metab. (2014) 40:1-14. doi: 10.1016/j.diabet.2013. 09.006

\section{CONCLUSION}

If POPs are a new risk factor for T2D, what can we do? POPs are different from traditional risk factors such as cigarette smoking which can be avoided through education, policy, etc. In modern society, complete avoidance of exposure to POPs is not possible due to the wide contamination of the food chain. Also, a large amount of POPs is already stored in our adipose tissue and they are continuously released to circulation. Furthermore, most problematic POPs such as OCPs or PCBs were already banned in most countries several decades ago.

Therefore, how to manage POPs in adipose tissue and circulation is the foremost issue in humans. Routine application of health behaviors such as exercise, calorie restriction, and high intake of phytochemicals have been suggested as practical ways to increase the continuous elimination of POPs from human bodies or mitigate harmful effects of POPs at the cellular level based on physiology of metabolism of xenobiotics and mitochondrial function $(51,52)$. Even though their effects need to be evaluated in randomized controlled trials, short-term clinical trials may fail to discover solid evidence due to the dynamic nature of serum concentrations of POPs. However, as these behaviors are currently well-accepted as healthy even without any consideration on POPs, they can be safely recommended to the public. Besides health behaviors, future research focusing on the development of more effective methods of eliminating POPs is needed.

\section{AUTHOR CONTRIBUTIONS}

Y-ML wrote the first draft of the manuscript. DJ contributed to the revision of draft. D-HL devised the main concept of manuscript and contributed to the revision of draft. All read and approved the submitted version.

\section{FUNDING}

This study was supported by the Kyungpook National University Research Fund, 2015 (No. 201514690000).

5. Ngwa EN, Kengne AP, Tiedeu-Atogho B, Mofo-Mato EP, Sobngwi E. Persistent organic pollutants as risk factors for type 2 diabetes. Diabetol Metab Syndr. (2015) 7:41. doi: 10.1186/s13098-015-0031-6

6. Jaacks LM, Staimez LR. Association of persistent organic pollutants and non-persistent pesticides with diabetes and diabetes-related health outcomes in Asia: a systematic review. Environ Int. (2015) 76:57-70. doi: 10.1016/j.envint.2014.12.001

7. Song Y, Chou EL, Baecker A, You NC, Song Y, Sun Q, et al. Endocrinedisrupting chemicals, risk of type 2 diabetes, and diabetes-related metabolic traits: a systematic review and meta-analysis. J Diabetes (2016) 8:516-32. doi: 10.1111/1753-0407.12325

8. Evangelou E, Ntritsos G, Chondrogiorgi M, Kavvoura FK, Hernandez AF, Ntzani EE, et al. Exposure to pesticides and diabetes: a systematic review and meta-analysis. Environ Int. (2016) 91:60-8. doi: 10.1016/j.envint.2016.02.013

9. Lind PM, Lind L. Endocrine-disrupting chemicals and risk of diabetes: an evidence-based review. Diabetologia (2018) 61:1495-502. doi: 10.1007/s00125-018-4621-3

10. Lee DH, Lee IK, Song K, Steffes M, Toscano W, Baker BA, et al. A strong dose-response relation between serum concentrations of persistent organic 
pollutants and diabetes: results from the National Health and Examination Survey 1999-2002. Diabetes Care (2006) 29:1638-44. doi: 10.2337/dc06-0543

11. Porta M, Gasull M, Puigdomenech E, Gari M, Bosch de Basea M, Guillen M, et al. Distribution of blood concentrations of persistent organic pollutants in a representative sample of the population of Catalonia. Environ Int. (2010) 36:655-64. doi: 10.1016/j.envint.2010.04.013

12. Birnbaum LS. The role of structure in the disposition of halogenated aromatic xenobiotics. Environ Health Perspect. (1985) 61:11-20.

13. American Diabetes Association. Diagnosis and classification of diabetes mellitus. Diabetes Care (2014) 37 (Suppl. 1):S81-90. doi: 10.2337/dc14-S081

14. Imamura F, Mukamal KJ, Meigs JB, Luchsinger JA, Ix JH, Siscovick DS, et al. Risk factors for type 2 diabetes mellitus preceded by beta-cell dysfunction, insulin resistance, or both in older adults: the cardiovascular health study. Am J Epidemiol. (2013) 177:1418-29. doi: 10.1093/aje/kws440

15. McCarthy MI. Genomics, type 2 diabetes, and obesity. N Engl J Med. (2010) 363:2339-50. doi: 10.1056/NEJMra0906948

16. Ma RC, Chan JC. Type 2 diabetes in East Asians: similarities and differences with populations in Europe and the United States. Ann N Y Acad Sci. (2013) 1281:64-91. doi: 10.1111/nyas.12098

17. Fabricio G, Malta A, Chango A, De Freitas Mathias PC. Environmental contaminants and pancreatic beta-cells. J Clin Res Pediatr Endocrinol. (2016) 8:257-63. doi: $10.4274 /$ jcrpe. 2812

18. Ruzzin J, Petersen R, Meugnier E, Madsen L, Lock EJ, Lillefosse H, et al. Persistent organic pollutant exposure leads to insulin resistance syndrome. Environ Health Perspect. (2010) 118:465-71. doi: 10.1289/ehp.0901321

19. Ibrahim MM, Fjaere E, Lock EJ, Naville D, Amlund H, Meugnier E, et al. Chronic consumption of farmed salmon containing persistent organic pollutants causes insulin resistance and obesity in mice. PLoS ONE (2011) 6:e25170. doi: 10.1371/journal.pone.0025170

20. Jensen TK, Timmermann AG, Rossing LI, Ried-Larsen M, Grontved A, Andersen LB, et al. Polychlorinated biphenyl exposure and glucose metabolism in 9-year-old Danish children. J Clin Endocrinol Metab. (2014) 99:E2643-51. doi: 10.1210/jc.2014-1683

21. Jorgensen ME, Borch-Johnsen K, Bjerregaard P. A cross-sectional study of the association between persistent organic pollutants and glucose intolerance among Greenland Inuit. Diabetologia (2008) 51:1416-22. doi: 10.1007/s00125-008-1066-0

22. Park SH, Ha E, Hong YS, Park H. Serum Levels of Persistent organic pollutants and insulin secretion among children Age 7-9 Years: A Prospective Cohort Study. Environ Health Perspect. (2016) 124:1924-30. doi: 10.1289/EHP147

23. Lee YM, Ha CM, Kim SA, Thoudam T, Yoon YR, Kim DJ, et al. Lowdose persistent organic pollutants impair insulin secretory function of pancreatic beta-cells: human and in vitro evidence. Diabetes (2017) 66:266980. doi: $10.2337 / \mathrm{db} 17-0188$

24. Morimoto A, Tatsumi Y, Deura K, Mizuno S, Ohno Y, Miyamatsu N, et al. Impact of impaired insulin secretion and insulin resistance on the incidence of type 2 diabetes mellitus in a Japanese population: the Saku study. Diabetologia (2013) 56:1671-9. doi: 10.1007/s00125-013-2932-y

25. Yoon KH, Lee JH, Kim JW, Cho JH, Choi YH, Ko SH, et al. Epidemic obesity and type 2 diabetes in Asia. Lancet (2006) 368:1681-8. doi: 10.1016/S0140-6736(06)69703-1

26. Chang AM, Halter JB. Aging and insulin secretion. Am J Physiol Endocrinol Metab. (2003) 284:E7-12. doi: 10.1152/ajpendo.00366.2002

27. Hue O, Marcotte J, Berrigan F, Simoneau M, Dore J, Marceau $\mathrm{P}$, et al. Plasma concentration of organochlorine compounds is associated with age and not obesity. Chemosphere (2007) 67:1463-7. doi: 10.1016/j.chemosphere.2006.10.033

28. Tanabe S, Minh TB. Dioxins and organohalogen contaminants in the Asia-Pacific region. Ecotoxicology (2010) 19:463-78. doi: 10.1007/s10646-009-0445-8

29. Tilg H, Moschen AR. Adipocytokines: mediators linking adipose tissue, inflammation and immunity. Nat Rev Immunol. (2006) 6:772-83. doi: $10.1038 /$ nri1937

30. Shulman GI. Ectopic fat in insulin resistance, dyslipidemia, and cardiometabolic disease. N Engl J Med. (2014) 371:2237-8. doi: 10.1056/NEJMc1412427

31. La Merrill M, Emond C, Kim MJ, Antignac JP, Le Bizec B, Clement $\mathrm{K}$, et al. Toxicological function of adipose tissue: focus on persistent organic pollutants. Environ Health Perspect. (2013) 121:162-9. doi: 10.1289/ehp. 1205485

32. Arsenescu V, Arsenescu RI, King V, Swanson H, Cassis LA. Polychlorinated biphenyl-77 induces adipocyte differentiation and proinflammatory adipokines and promotes obesity and atherosclerosis. Environ Health Perspect. (2008) 116:761-8. doi: 10.1289/ehp.10554

33. May P, Bremond P, Sauzet C, Piccerelle P, Grimaldi F, Champion S, et al. In vitro cocktail effects of PCB-DL (PCB118) and Bulky PCB (PCB153) with $\mathrm{BaP}$ on Adipogenesis and on expression of genes involved in the establishment of a pro-inflammatory state. Int J Mol Sci. (2018) 19:E841. doi: 10.3390/ijms19030841

34. Pestana D, Teixeira D, Meireles M, Marques C, Norberto S, Sa C, et al. Adipose tissue dysfunction as a central mechanism leading to dysmetabolic obesity triggered by chronic exposure to p,p'-DDE. Sci Rep. (2017) 7:2738. doi: 10.1038/s41598-017-02885-9

35. Cinti S, Mitchell G, Barbatelli G, Murano I, Ceresi E, Faloia E, et al. Adipocyte death defines macrophage localization and function in adipose tissue of obese mice and humans. J Lipid Res. (2005) 46:2347-55. doi: 10.1194/jlr.M500294-JLR200

36. Rosen ED, Spiegelman BM. Adipocytes as regulators of energy balance and glucose homeostasis. Nature (2006) 444:847-53. doi: 10.1038/nature 05483

37. de Ferranti S, Mozaffarian D. The perfect storm: obesity, adipocyte dysfunction, and metabolic consequences. Clin Chem. (2008) 54:945-55. doi: 10.1373/clinchem.2007.100156

38. Morigny P, Houssier M, Mouisel E, Langin D. Adipocyte lipolysis and insulin resistance. Biochimie (2016) 125:259-66. doi: 10.1016/j.biochi.2015.10.024

39. Louis C, Tinant G, Mignolet E, Thome JP, Debier C. PCB-153 shows different dynamics of mobilisation from differentiated rat adipocytes during lipolysis in comparison with PCB-28 and PCB-118. PLoS ONE (2014) 9:e106495. doi: 10.1371/journal.pone. 0106495

40. Jandacek RJ, Anderson N, Liu M, Zheng S, Yang Q, Tso P. Effects of yo-yo diet, caloric restriction, and olestra on tissue distribution of hexachlorobenzene. Am J Physiol Gastrointest Liver Physiol. (2005) 288:G2929. doi: 10.1152/ajpgi.00285.2004

41. Ahotupa M, Mantyla E. Adipose tissue content as a modifier of the tissue distribution, biological effects, and excretion of a hexachlorobiphenyl in C57BL/6J and DBA/JBOMf mice. Mol Pharmacol. (1983) 24:464-70.

42. Antonopoulos AS, Oikonomou EK, Antoniades C, Tousoulis D. From the BMI paradox to the obesity paradox: the obesity-mortality association in coronary heart disease. Obes Rev. (2016) 17:989-1000. doi: 10.1111/obr.12440

43. Hainer V, Aldhoon-Hainerova I. Obesity paradox does exist. Diabetes Care (2013) 36 (Suppl. 2):S276-81. doi: 10.2337/dcS13-2023

44. Hong NS, Kim KS, Lee IK, Lind PM, Lind L, Jacobs DR, et al. The association between obesity and mortality in the elderly differs by serum concentrations of persistent organic pollutants: a possible explanation for the obesity paradox. Int J Obes. (2012) 36:1170-5. doi: 10.1038/ijo.2011.187

45. Imbeault P, Tremblay A, Simoneau JA, Joanisse DR. Weight loss-induced rise in plasma pollutant is associated with reduced skeletal muscle oxidative capacity. Am J Physiol Endocrinol Metab. (2002) 282:E574-9. doi: 10.1152/ajpendo.00394.2001

46. Jansen A, Lyche JL, Polder A, Aaseth J, Skaug MA. Increased blood levels of persistent organic pollutants (POP) in obese individuals after weight loss-A review. J Toxicol Environ Health B Crit Rev. (2017) 20:22-37. doi: 10.1080/10937404.2016.1246391

47. Lim JS, Son HK, Park SK, Jacobs DR Jr, Lee DH. Inverse associations between long-term weight change and serum concentrations of persistent organic pollutants. Int J Obes. (2011) 35:744-7. doi: 10.1038/ijo.2010.188

48. Pelletier C, Doucet E, Imbeault P, Tremblay A. Associations between weight loss-induced changes in plasma organochlorine concentrations, serum $\mathrm{T}_{3}$ concentration, and resting metabolic rate. Toxicol Sci. (2002) 67:46-51. doi: $10.1093 /$ toxsci/67.1.46

49. Tremblay A, Pelletier C, Doucet E, Imbeault P. Thermogenesis and weight loss in obese individuals: a primary association with organochlorine pollution. Int J Obes Relat Metab Disord. (2004) 28:936-9. doi: 10.1038/sj.ijo.0802527

50. Look ARG, Wing RR, Bolin P, Brancati FL, Bray GA, Clark JM, et al. Cardiovascular effects of intensive lifestyle intervention in type 2 diabetes. $N$ Engl J Med. (2013) 369:145-54. doi: 10.1056/NEJMoa1212914 
51. Lee YM, Kim KS, Jacobs DR Jr, Lee DH. Persistent organic pollutants in adipose tissue should be considered in obesity research. Obes Rev. (2017) 18:129-39. doi: 10.1111/obr.12481

52. Kim SA, Lee YM, Choi JY, Jacobs DR Jr, Lee DH. Evolutionarily adapted hormesis-inducing stressors can be a practical solution to mitigate harmful effects of chronic exposure to low dose chemical mixtures. Environ Pollut. (2017) 233:725-34. doi: 10.1016/j.envpol.2017.10.124

53. McLachlan AJ, Pont LG. Drug metabolism in older people-a key consideration in achieving optimal outcomes with medicines. J Gerontol A Biol Sci Med Sci. (2012) 67:175-80. doi: 10.1093/gerona/glr118

54. Lee DH, Porta M, Lind L, Lind PM, Jacobs DR Jr. Neurotoxic chemicals in adipose tissue: A role in puzzling findings on obesity and dementia. Neurology (2018) 90:176-82. doi: 10.1212/WNL.0000000000004851

55. Chamorro-Garcia R, Blumberg B. Transgenerational effects of obesogens and the obesity epidemic. Curr Opin Pharmacol. (2014) 19:153-8. doi: 10.1016/j.coph.2014.10.010

56. Janesick AS, Shioda T, Blumberg B. Transgenerational inheritance of prenatal obesogen exposure. Mol Cell Endocrinol. (2014) 398:31-5. doi: 10.1016/j.mce.2014.09.002

57. Heindel JJ, Newbold R, Schug TT. Endocrine disruptors and obesity. Nat Rev Endocrinol. (2015) 11:653-61. doi: 10.1038/nrendo.2015.163

58. Thayer KA, Heindel JJ, Bucher JR, Gallo MA. Role of environmental chemicals in diabetes and obesity: a National Toxicology Program workshop review. Environ Health Perspect. (2012) 120:779-89. doi: 10.1289/ehp.1104597

59. Kloting N, Fasshauer M, Dietrich A, Kovacs P, Schon MR, Kern M, et al. Insulin-sensitive obesity. Am J Physiol Endocrinol Metab. (2010) 299:E506-15. doi: 10.1152/ajpendo.00586.2009

60. Cano-Sancho G, Salmon AG, La Merrill MA. Association between Exposure to p,p'-DDT and Its Metabolite p,p'-DDE with Obesity: integrated systematic review and meta-analysis. Environ Health Perspect. (2017) 125:096002. doi: $10.1289 /$ EHP527

61. Alexander DL, Ganem LG, Fernandez-Salguero P, Gonzalez F, Jefcoate CR. Aryl-hydrocarbon receptor is an inhibitory regulator of lipid synthesis and of commitment to adipogenesis. J Cell Sci. (1998) 111 ( Pt 22):3311-22.

62. Bowe B, Xie Y, Li T, Yan Y, Xian H, Al-Aly Z. The 2016 global and national burden of diabetes mellitus attributable to $\mathrm{PM}_{2.5}$ air pollution. Lancet Planet Health (2018) 2:e301-12. doi: 10.1016/S2542-5196(18)30140-2

63. Lanphear BP. Low-level toxicity of chemicals: no acceptable levels? PLoS Biol. (2017) 15:e2003066. doi: 10.1371/journal.pbio.2003066

64. Lanphear BP, Rauch S, Auinger P, Allen RW, Hornung RW. Low-level lead exposure and mortality in US adults: a population-based cohort study. Lancet Public Health (2018) 3:e177-e84. doi: 10.1016/S2468-2667(18)30025-2

65. Banner W, Kahn CM. Low blood lead level effects on intelligence: can a doseresponse curve be determined from the epidemiological data? Clin Toxicol. (2014) 52:113-7. doi: 10.3109/15563650.2013.876544

66. Bowers TS, Beck BD. What is the meaning of non-linear dose-response relationships between blood lead concentrations and IQ? Neurotoxicology (2006) 27:520-4. doi: 10.1016/j.neuro.2006.02.001

67. Vandenberg LN, Colborn T, Hayes TB, Heindel JJ, Jacobs DR Jr, Lee $\mathrm{DH}$, et al. Hormones and endocrine-disrupting chemicals: low-dose effects and nonmonotonic dose responses. Endocr Rev. (2012) 33:378-455. doi: 10.1210/er.2011-1050

68. Calabrese EJ, Mattson MP. How does hormesis impact biology, toxicology, and medicine? NPJ Aging Mech Dis. (2017) 3:13. doi: 10.1038/s41514-017-0013-z

69. Lee DH, Jacobs DR Jr. Hormesis and public health: can glutathione depletion and mitochondrial dysfunction due to very low-dose chronic exposure to persistent organic pollutants be mitigated? J Epidemiol Community Health (2015) 69:294-300. doi: 10.1136/jech-2014-203861

70. Kortenkamp A. Low dose mixture effects of endocrine disrupters and their implications for regulatory thresholds in chemical risk assessment. Curr Opin Pharmacol. (2014) 19:105-11. doi: 10.1016/j.coph.2014.08.006
71. Carnevali O, Notarstefano V, Olivotto I, Graziano M, Gallo P, Di Marco Pisciottano I, et al. Dietary administration of EDC mixtures: a focus on fish lipid metabolism. Aquat Toxicol. (2017) 185:95-104. doi: 10.1016/j.aquatox.2017.02.007

72. Hu Y, Wang R, Xiang Z, Qian W, Han X, Li D. Antagonistic effects of a mixture of low-dose nonylphenol and di-n-butyl phthalate (monobutyl phthalate) on the Sertoli cells and serum reproductive hormones in prepubertal male rats in vitro and in vivo. PLoS ONE (2014) 9:e93425. doi: 10.1371/journal.pone.0093425

73. Ribeiro E, Ladeira C, Viegas S. EDCs mixtures: a stealthy hazard for human health? Toxics (2017) 5:5. doi: 10.3390/toxics5010005

74. Sarria MP, Santos MM, Reis-Henriques MA, Vieira NM, Monteiro NM. The unpredictable effects of mixtures of androgenic and estrogenic chemicals on fish early life. Environ Int. (2011) 37:418-24. doi: 10.1016/j.envint.2010.11.004

75. Meyer JN, Leung MC, Rooney JP, Sendoel A, Hengartner MO, Kisby GE, et al. Mitochondria as a target of environmental toxicants. Toxicol Sci. (2013) 134:1-17. doi: 10.1093/toxsci/kft102

76. Lim S, Ahn SY, Song IC, Chung MH, Jang HC, Park KS, et al. Chronic exposure to the herbicide, atrazine, causes mitochondrial dysfunction and insulin resistance. PLoS ONE (2009) 4:e5186. doi: 10.1371/journal.pone.0005186

77. Liu Q, Wang Q, Xu C, Shao W, Zhang C, Liu H, et al. Organochloride pesticides impaired mitochondrial function in hepatocytes and aggravated disorders of fatty acid metabolism. Sci Rep. (2017) 7:46339. doi: 10.1038/srep46339

78. Mailloux RJ, Florian M, Chen Q, Yan J, Petrov I, Coughlan MC, et al. Exposure to a northern contaminant mixture (NCM) alters hepatic energy and lipid metabolism exacerbating hepatic steatosis in obese JCR rats. PLoS ONE (2014) 9:e106832. doi: 10.1371/journal.pone.0106832

79. Byczkowski JZ. The mode of action of $\mathrm{p}, \mathrm{p}^{\prime}=\mathrm{DDT}$ on mammalian mitochondria. Toxicology (1976) 6:309-14.

80. Miller WL. Steroid hormone synthesis in mitochondria. Mol Cell Endocrinol. (2013) 379:62-73. doi: 10.1016/j.mce.2013.04.014

81. Chan DC. Mitochondria: dynamic organelles in disease, aging, and development. Cell (2006) 125:1241-52. doi: 10.1016/j.cell.2006.06.010

82. Sano M, Fukuda K. Activation of mitochondrial biogenesis by hormesis. Circ Res. (2008) 103:1191-3. doi: 10.1161/CIRCRESAHA.108.189092

83. Yun J, Finkel T. Mitohormesis. Cell Metab. (2014) 19:757-66. doi: 10.1016/j.cmet.2014.01.011

84. Merry TL, Ristow M. Mitohormesis in exercise training. Free Radic Biol Med. (2016) 98:123-30. doi: 10.1016/j.freeradbiomed.2015.11.032

85. Ristow M, Zarse K. How increased oxidative stress promotes longevity and metabolic health: the concept of mitochondrial hormesis (mitohormesis). Exp Gerontol. (2010) 45:410-8. doi: 10.1016/j.exger.2010.03.014

86. Grabacka MM, Gawin M, Pierzchalska M. Phytochemical modulators of mitochondria: the search for chemopreventive agents and supportive therapeutics. Pharmaceuticals (2014) 7:913-42. doi: 10.3390/ph 7090913

87. Lee DH, Jacobs DR Jr. Methodological issues in human studies of endocrine disrupting chemicals. Rev Endocr Metab Disord. (2015) 16:289-97. doi: $10.1007 / \mathrm{s} 11154-016-9340-9$

Conflict of Interest Statement: The authors declare that the research was conducted in the absence of any commercial or financial relationships that could be construed as a potential conflict of interest.

Copyright (c) 2018 Lee, Jacobs and Lee. This is an open-access article distributed under the terms of the Creative Commons Attribution License (CC BY). The use, distribution or reproduction in other forums is permitted, provided the original author(s) and the copyright owner(s) are credited and that the original publication in this journal is cited, in accordance with accepted academic practice. No use, distribution or reproduction is permitted which does not comply with these terms. 